\title{
Stationary Points for Multifunctions on Two Complete Metric Spaces
}

\author{
VALERIU POPA
}

\begin{abstract}
In this paper we prove a general fixed point theorem for multifunctions on two complete metric spaces which generalizes the main results from [2] and [5].
\end{abstract}

\section{INTRODUCTION}

Let $(X, d)$ be a complete metric space and let $B(X)$ be the set of all nonempty subsets of $X$. As in [1] we define the function $\delta(A, B)$ with $A$ and $B$ in $B(X)$ by $\delta(A, B)=\sup \{d(a, b): a \in A, b \in B\}$.

If $A$ is consists of a single point we write $\delta(A, B)=\delta(a, B)$. If $B$ also consists of single point $b$ then $\delta(a, b)=d(a, b)$. It follows immediately that: $\delta(A, B)=$ $\delta(B, A) \geq 0$ and $\delta(A, B) \leq \delta(A, C)+\delta(C, B)$ for $A, B, C$ in $B(X)$. If $\delta(A, B)=0$ then $A=B=\{a\}$.

Now if $\left\{A_{n}: n=1,2, \ldots\right\}$ is a sequence in $B(X)$, we say that it converges to the set $A$ in $B(X)$ if:

(i) each point $a \in A$ is limit of some convergent sequence $\left\{a_{n} \in A_{n}: n=\right.$ $1,2, \ldots\}$

(ii) for arbitrary $\epsilon>0$, there exists an integer $N$ such that $A_{n} \subset A_{\epsilon}$ for $n>N$, where $A_{\epsilon}$ is the union of all open spheres with centers in $A$ of radius $\epsilon$.

The set $A$ is said to be limit of the sequence $\left\{A_{n}\right\}$.

The following Lemma was proved in [1].

Lemma 1.1. If $\left\{A_{n}\right\}$ and $\left\{B_{n}\right\}$ are sequences of bounded subsets of a complete metric space $(X, d)$ which converges to the bounded subsets $A$ and $B$, respectively, then the sequence $\left\{\delta\left(A_{n}, B_{n}\right)\right\}$ converges to $\delta(A, B)$.

Let $T$ be a multifunction of $X$ into $B(X) . \quad \mathrm{z}$ is a stationary point of $T$ if $T z=\{z\}$.

In 1981, Fisher [2] initiated the study of fixed points on two metric spaces. In 1991, the present author [5] proved other theorems on two metric spaces.

2000 Mathematics Subject Classification. Primary 54H25.

Key words and phrases. fixed point, multifunction, implicit relation, complete metric space. 
The following fixed points theorems are proved in [2], resp. [5].

Theorem $1.1([2])$. . Let $(X, d)$ and $(Y, \rho)$ be complete metric spaces. If $T$ is a mapping of $X$ into $Y$ and $S$ is a mapping of $Y$ into $X$ satisfying the inequalities

$$
\begin{aligned}
& \rho(T x, T S y) \leq c \max \{d(x, S y), \rho(y, T x), \rho(y, T S y)\}, \\
& d(S y, S T x) \leq c \max \{\rho(y, T x), d(x, S y), d(x, S T x)\}
\end{aligned}
$$

for all $x$ in $X$ and $y$ in $Y$, where $0 \leq c<1$, then $S T$ has a unique fixed point $z$ in $X$ and $T S$ has a unique fixed point $w$ in $Y$. Further, $T z=w$ and $S w=z$.

Theorem $1.2([5])$. Let $(X, d)$ and $(Y, e)$ be complete metric spaces. If $T$ is a mapping of $X$ into $Y$ and $S$ is a mapping of $Y$ into $X$ satisfying the inequalities

$$
\begin{aligned}
& e^{2}(T x, T S y) \leq c_{1} \max \{d(x, S y) e(y, T x), d(x, S y) e(y, T S y), e(y, T x) e(y, T S y)\}, \\
& d^{2}(S y, S T x) \leq c_{2} \max \{e(y, T x) d(x, S y), e(y, T x) d(x, S T x), d(x, S y) d(x, S T x)\}
\end{aligned}
$$

for all $x$ in $X$ and $y$ in $Y$, where $0 \leq c_{1}, c_{2}<1$, then $S T$ has a unique fixed point $z$ in $X$ and $T S$ has a unique fixed point $w$ in $Y$. Furthermore, $T z=w$ and $S w=z$.

Recently, some fixed points theorems for multifunctions on two complete metric spaces have been proved in [3], [4], [6].

In this paper we prove two generalizations of Theorems 1 and 2 for single valued and set valued mappings satisfying two implicit relations.

\section{IMPLICIT RELATIONS}

Let $\mathcal{F}_{4}$ be the set of all continuous functions $F: R_{+}^{4} \rightarrow R$ such that:

$\left(F_{1}\right): F$ is nonincreasing in variables $t_{2}, t_{3}$;

$\left(F_{2}\right)$ : there exists $h \in[0,1)$ such that for every $u \geq 0, v \geq 0$ with:

a) $F(u, 0, u, v) \leq 0$ or $\quad$ b) $F(u, u, 0, v) \leq 0$

we have $u \leq h v$.

Example 2.1. $F\left(t_{1}, \ldots, t_{4}\right)=t_{1}-k \max \left\{t_{2}, t_{3}, t_{4}\right\}$ where $k \in[0,1)$.

$\left(F_{1}\right)$ : Obviously.

$\left(F_{2}\right)$ : Let $u>0$ and $F(u, 0, u, v)=u-k \max \{u, v\} \leq 0$.

If $u \geq v$ then $u(1-k) \leq 0$, a contradiction.

Thus $u<v$ and $u \leq h v$. Similarly, $F(u, u, 0, v) \leq 0$ implies $u \leq h v$. If $u=0$, then $u \leq h v$.

Example 2.2. $F\left(t_{1}, \ldots, t_{4}\right)=t_{1}^{2}-c \max \left\{t_{2} t_{4}, t_{2} t_{3}, t_{3} t_{4}\right\}$ where $c \in[0,1)$.

$\left(F_{1}\right)$ : Obviously.

$\left(F_{2}\right)$ : Let $u>0$ and $F(u, 0, u, v)=u^{2}-c u v \leq 0$, which implies $u \leq h v$, where $h=c \in[0,1)$.

Similarly, $F(u, u, 0, v) \leq 0$ implies $u \leq h v$.

If $u=0$, then $u \leq h v$. 
Example 2.3. $F\left(t_{1}, \ldots, t_{4}\right)=t_{1}^{3}-\left(a t_{1}^{2} t_{2}+b t_{3}^{3}+c t_{4}^{3}\right)$ where $a, b, c>0$ and $a+b+c<1$.

$\left(F_{1}\right)$ : Obviously.

$\left(F_{2}\right): F(u, 0, u, v)=u^{3}-\left[b u^{3}+c v^{3}\right] \leq 0$ implies $u \leq h_{1} v$, where $h_{1}=$ $\left(\frac{c}{1-b}\right)^{\frac{1}{3}}<1$.

Similarly, $F(u, u, 0, v) \leq 0$ implies $u \leq h_{2} v$, where $h_{2}=\left(\frac{c}{1-a}\right)^{\frac{1}{3}}<1$. Let $h=\max \left\{h_{1}, h_{2}\right\}$, then $u \leq h v$.

Example 2.4. $F\left(t_{1}, \ldots, t_{4}\right)=t_{1}-c \frac{t_{2}+t_{3}+t_{4}}{1+t_{4}}$ where $0 \leq c<\frac{1}{2}$

$\left(F_{1}\right)$ : Obviously.

$\left(F_{2}\right): F(u, 0, u, v)=u-c \frac{u+v}{1+v}$ implies $u-c(u+v) \leq 0$ and $u \leq h v$, where $h=\frac{c}{1-c}<1$. Similarly, $F(u, u, 0, v) \leq 0$ implies $u \leq h v$.

\section{MAIN RESUltS}

Theorem 3.1. Let $\left(X, d_{1}\right)$ and $\left(Y, d_{2}\right)$ be two complete metric spaces and let $F$ be a mapping of $X$ into $B(Y)$ and let $G$ be a mapping of $Y$ into $B(X)$ satisfying the inequalities:

$$
\begin{aligned}
& \Phi_{1}\left(\delta_{1}(G F x, G y), d_{1}(x, G y), \delta_{1}(x, G F x), \delta_{2}(y, F x)\right) \leq 0 \\
& \Phi_{2}\left(\delta_{2}(F G y, F x), d_{2}(y, F x), \delta_{2}(y, F G y), \delta_{1}(x, G y)\right) \leq 0
\end{aligned}
$$

for all $x$ in $X$ and $y$ in $Y$, where $\Phi_{1}, \Phi_{2} \in \mathcal{F}_{4}$, then $G F$ has a stationary point $z$ in $X$ and $F G$ has a stationary point $w$ in $Y$. Furthermore, $F z=\{w\}$ and $G w=\{z\}$.

Proof. Let $x_{1}$ be an arbitrary point in $X$. Define sequences $\left\{x_{n}\right\}$ and $\left\{y_{n}\right\}$ in $X$ and $Y$, respectively, as follows: choose a point $y_{1}$ in $F x_{1}$ and a point $x_{2}$ in $G y_{1}$. In general, having chosen $x_{n}$ in $X$ and $y_{n}$ in $Y$, we choose $x_{n+1}$ in $G y_{n}$ and then $y_{n+1}$ in $F x_{n+1}$ for $n=1,2, \ldots$

Then, by (1), we have successively

$$
\begin{array}{r}
\Phi_{1}\left(\delta_{1}\left(G F x_{n+1}, G y_{n}\right), d_{1}\left(x_{n+1}, G y_{n}\right), \delta_{1}\left(x_{n+1}, G F x_{n+1}\right), \delta_{2}\left(y_{n}, F x_{n+1}\right)\right) \leq 0 \\
\Phi_{1}\left(\delta_{1}\left(G F x_{n+1}, G y_{n}\right), 0, \delta_{1}\left(G y_{n}, G F x_{n+1}\right), \delta_{2}\left(y_{n}, F x_{n+1}\right)\right) \leq 0
\end{array}
$$

which implies

$$
\delta_{1}\left(G F x_{n+1}, G y_{n}\right) \leq h \delta_{2}\left(y_{n}, F x_{n+1}\right) .
$$

By (2) we have successively

$$
\begin{array}{r}
\Phi_{2}\left(\delta_{2}\left(F G y_{n}, F x_{n}\right), d_{2}\left(y_{n}, F x_{n}\right), \delta_{2}\left(y_{n}, F G y_{n}\right), \delta_{1}\left(x_{n}, G y_{n}\right)\right) \leq 0 \\
\Phi_{2}\left(\delta_{2}\left(F G y_{n}, F x_{n}\right), 0, \delta_{2}\left(F x_{n}, F G y_{n}\right), \delta_{1}\left(x_{n}, G y_{n}\right)\right) \leq 0
\end{array}
$$

which implies

$$
\delta_{2}\left(F G y_{n}, F x_{n}\right) \leq h_{2} \delta_{1}\left(x_{n}, G y_{n}\right) .
$$


Thus, it follows from (3) and (4) that

$$
\begin{aligned}
d_{1}\left(x_{n+1}, x_{n+2}\right) & \leq \delta_{1}\left(G y_{n}, G F x_{n+1}\right) \leq h_{1} \delta_{2}\left(y_{n}, F x_{n+1}\right) \leq h_{1} \delta_{2}\left(F x_{n}, G F y_{n}\right) \leq \\
& \leq h_{1} h_{2} \delta_{1}\left(x_{n}, G y_{n}\right) \leq \ldots\left(h_{1} h_{2}\right)^{n} \delta_{1}\left(x_{1}, G F x_{1}\right) .
\end{aligned}
$$

Similarly, we can prove that

$$
d_{2}\left(y_{n+1}, y_{n}\right) \leq\left(h_{1} h_{2}\right)^{n} \delta_{2}\left(y_{1}, F G y_{1}\right) .
$$

Now, it follows that for $n=1,2, \ldots$ and $r \in N^{*}$

$$
\begin{aligned}
d_{1}\left(x_{n+1}, x_{n+r+1}\right) \leq & \delta_{1}\left(G y_{n}, G F x_{n+r}\right) \leq \\
\leq & \delta_{1}\left(G y_{n}, G y_{n+1}\right)+\delta_{1}\left(G y_{n+1}, y_{n+2}\right)+\cdots+ \\
& +\delta_{1}\left(G y_{n+r-1}, G F x_{n+r}\right) \leq \\
\leq & \delta_{1}\left(G y_{n}, G F x_{n+1}\right)+\delta_{1}\left(G y_{n+1}, G F x_{n+2}\right)+\cdots+ \\
& +\delta_{1}\left(G y_{n+r-1}, G F x_{n+r}\right) \leq \\
\leq & \left\{\left(h_{1} h_{2}\right)^{n}+\left(h_{1} h_{2}\right)^{n+1}+\cdots+\left(h_{1} h_{2}\right)^{n+r-1}\right\} \delta_{1}\left(x_{1}, G F x_{1}\right)<\epsilon
\end{aligned}
$$

for $n$ greater than some $N$ since $h_{1} h_{2}<1$.

Therefore, the sequence $\left\{x_{n}\right\}$ is a Cauchy sequence in the complete metric space $X$ and so it has a limit $z$ in $X$.

Similarly, the sequence $\left\{y_{n}\right\}$ is a Cauchy sequence in the complete metric space $Y$ and so it has a limit $w$ in $Y$.

Further

$$
\begin{aligned}
\delta_{1}\left(z, G F x_{n}\right) & \leq d_{1}\left(z, x_{m+1}\right)+\delta_{1}\left(x_{m+1}, G F x_{n}\right) \leq \\
& \leq d_{1}\left(z, x_{m+1}\right)+\delta_{1}\left(G y_{m}, G F x_{n}\right) \\
& \leq d_{1}\left(z, x_{m+1}\right)+\epsilon \text { for } m, n>N .
\end{aligned}
$$

Letting $m$ tend to infinity it follows that

$$
\delta_{1}\left(z, G F x_{n}\right)<\epsilon
$$

for $n>N$ and

$$
\lim G F x_{n}=z=\lim G y_{n} .
$$

Similarly,

$$
\lim F G y_{n}=w=\lim F x_{n} .
$$

Using inequality $(2)$ and $\left(F_{1}\right)$ we have

$$
\Phi_{2}\left(\delta_{2}\left(F G y_{n}, F z\right), \delta_{2}\left(y_{n}, F z\right), \delta_{2}\left(y_{n}, F G y_{n}\right), \delta_{1}\left(z, G y_{n}\right)\right) \leq 0 .
$$

Letting $n$ tend to infinity we obtain successively

$$
\begin{aligned}
\Phi_{2}\left(\delta_{2}(w, F z), \delta_{2}(w, F z), \delta_{2}(w, w), \delta_{1}(z, z)\right) & \leq 0 \\
\Phi_{2}\left(\delta_{2}(w, F z), \delta_{2}(w, F z), 0,0\right) & \leq 0
\end{aligned}
$$

which implies $\delta_{2}(w, F z)=0$. Thus

$$
F z=\{w\} .
$$


Similarly, we can prove that

$$
G w=\{z\}
$$

From (7) and (8), it follows that

$$
G F z=G w=\{z\} \text { and } F G w=F z=\{w\} .
$$

Thus $z$ is a stationary point of $G F$ and $w$ is a stationary point of $F G$. This completes the proof of Theorem 3.

Theorem 3.2. Let $\left(X, d_{1}\right)$ and $\left(Y, d_{2}\right)$ be two complete metric spaces and let $f$ be a single valued mapping of $X$ into $Y$ and $g$ a single valued mapping of $Y$ into $X$ satisfying the inequalities

$$
\begin{aligned}
& \Phi_{1}\left(d_{1}(g f x, g y), d_{1}(x, g y), d_{1}(x, g f x), d_{2}(y, f x)\right) \leq 0 \\
& \Phi_{2}\left(d_{2}(f g y, f x), d_{2}(y, f x), d_{2}(y, f g y), d_{1}(x, g y)\right) \leq 0
\end{aligned}
$$

for all $x$ in $X$ and $y$ in $Y$, where $\Phi_{1}, \Phi_{2} \in \mathcal{F}_{4}$.

Then $g f$ has an unique fixed point $z$ in $X$ and $f g$ has an unique fixed point $w$ in $Y$. Further, $f z=w$ and $g w=z$.

Proof. The existence of $z$ and $w$ follows from Theorem 3. Now suppose that $g f$ has a second fixed point $z^{\prime}$.

Then by $\left(1^{\prime}\right)$ we have successively

$$
\begin{array}{r}
\Phi_{1}\left(d_{1}\left(g f z, g f z^{\prime}\right), d_{1}\left(z, g f z^{\prime}\right), d_{1}(z, g f z), d_{2}\left(f z^{\prime}, f z\right)\right) \leq 0 \\
\Phi_{1}\left(d_{1}\left(z, z^{\prime}\right), d\left(z, z^{\prime}\right), 0, d\left(f z, f z^{\prime}\right)\right) \leq 0
\end{array}
$$

which implies

$$
d\left(z, z^{\prime}\right) \leq h_{1} d\left(f z, f z^{\prime}\right) .
$$

Similarly, by $\left(2^{\prime}\right)$ we have successively

$$
\begin{array}{r}
\Phi_{2}\left(d_{2}\left(f g f z, f z^{\prime}\right), d_{2}\left(f z, f z^{\prime}\right), d_{2}(f z, f g f z), d_{1}\left(z^{\prime}, g f z\right)\right) \leq 0 \\
\Phi_{2}\left(d_{2}\left(f z, f z^{\prime}\right), d\left(f z, f z^{\prime}\right), 0, d\left(z, z^{\prime}\right)\right) \leq 0
\end{array}
$$

which implies

$$
d_{2}\left(f z, f z^{\prime}\right) \leq h_{2} d\left(z, z^{\prime}\right) .
$$

By (9) and (10) we have

$$
d_{1}\left(z, z_{1}\right) \leq h_{1} d_{2}\left(f z, f z^{\prime}\right) \leq\left(h_{1} h_{2}\right) d_{1}\left(z, z^{\prime}\right) .
$$

Since $h_{1} h_{2}<1$ it follows that $z=z^{\prime}$.

Similarly $f g$ has a unique fixed point.

Corollary 3.1. Theorem 1.1.

Proof. The proof follows from Theorem 3.2 and Example 1.

Corollary 3.2. Theorem 1.2.

Proof. The proof follows from Theorem 3.2 and Example 2. 


\section{REFERENCES}

[1] Fisher, B., Common fixed points of mappings and set valued mappings, Rostok Math.Kolloq. 8(1981), 68-77.

[2] Fisher, B., Fixed points on two metric spaces, Glasnik Mat. 16(36),(1981), 333-337.

[3] Fisher, B. and Türköglu,D., Related fixed point for set valued mappings on two metric spaces, Internat. J.Math.Math.Sci. 23(2000), 205-210.

[4] Liu Z., Liu Q., Stationary points for set-valued mappings on two metric spaces, Rostok Math. Kolloq. 55(2001), 23-29.

[5] Popa V., Fixed points on two complete metric spaces, Zb.Rad.Prirod.Mat.Fiz. Ser.Mat.Univ. Novom Sadu, 21(1991),83-93.

[6] Popa V., Two general fixed point theorem for mappings on two metric spaces, Anal.Univ.Galaţi, fasc.II. Mat.Fiz.Mec.Teor.Suppl. vol.18(23)(2001),19-24.

Department of Mathematics

UNIVERSITY OF BACĂU

5500 BACĂU, ROMANIA

E-mail address: vpopa@ub.ro 